\begin{tabular}{|l|l|}
\hline Postprint Version & 1.0 \\
\hline Journal website & http://www3.interscience.wiley.com/journal/119188484/abstract \\
\hline Pubmed link & http://www.ncbi.nlm.nih.gov/pubmed/11111610 \\
\hline DOI & $10.1046 /$ j.1365-2702.2000.00356.x \\
\hline
\end{tabular}

\title{
A randomized trial of a Telephone Reassurance Programme for patients recently discharged from an ophthalmic unit
}

\author{
HAN BOTER MSN, RN, PATRIEK MISTIAEN MSN, RN \& INGRID GROENEWEGEN RN \\ ${ }^{1}$ Nurse Researcher, Research Centre Primary-Secondary Health Care, University Hospital Vrije \\ Universiteit, Amsterdam, The Netherlands, \\ ${ }^{2}$ Nurse Researcher, Research Centre Primary-Secondary Health Care, University Hospital Vrije \\ Universiteit, Amsterdam, The Netherlands, \\ ${ }^{3}$ Head Nurse, Ophthalmic Unit, University Hospital Vrije Universiteit, Amsterdam, The Netherlands
}

\begin{abstract}
- Patients often experience problems after discharge, for instance with housekeeping or a general lack of information.

- The effect of a nurse-initiated Telephone Reassurance Programme (TRP) on ophthalmic patient outcomes was investigated.

- Patients in the intervention group were phoned by a nurse 3-6 days after being randomized and discharged home.

- Patients in both intervention and control groups received a questionnaire 1 week and 1 month after discharge to assess the patient outcomes 'Informational needs', 'Uncertainty', 'Emotional complaints' and 'Functional limitations'.

- In an attempt to explain the lack of statistically significant results, the limitations related to the participants, intervention and outcomes are discussed.
\end{abstract}

\section{INTRODUCTION}

Patients, including ophthalmic patients, are at risk of being discharged without adequate preparation for self-management at home. This is partly the result of medical and technological developments and financial constraints that restrict the period of hospitalization, and the time available for making adequate and comprehensive discharge preparations. In addition, patients do not always ask the necessary questions when they are in a hospital ( Van Beelen, 1996), or they forget the information they have been given ( Tierney et al., 1994 ). Another difficulty is that it is sometimes hard to estimate the extent to which a patient will be self-supporting after discharge ( Arenth \& Mamon, 1985).

Although nurses try to prepare patients adequately for discharge, they do not always succeed. A number of studies indicate that some patients do, indeed, have problems after discharge. For example, the results of a study among 145 elderly patients who had recently been discharged indicated that $79 \%$ of the patients did not feel that they had been adequately informed, $77 \%$ had difficulties with housekeeping and almost $40 \%$ had one or more unmet needs ( Mistiaen et al., 1997 ). Other studies also found that postdischarge problems existed: concerns related to the patient's progress and how much activity is good (Boyle et al., 1992 ), whilst unmet needs related to treatment, activities and other aspects of self-sufficiency ( Mamon et al., 1992 ), informational needs ( McWilliam \& Sangster, 1994), financial concerns, family/relationship problems ( 
North et al., 1991 ), and difficulties related to going out, sleeping, taking medicine, washing and bathing, pain, fatigue and feeling ill ( Tierney et al., 1994 ). Comparable results are also found among ophthalmic patients ( Smith \& Drance, 1984; Allen \& Oberle, 1993; Law, 1997; Boter et al., 1998 b).

Care institutions try to address postdischarge problems in several ways: for example by a home care preoperative teaching programme ( Allen et al., 1992 ), a transitional home follow-up by nurse specialists ( Brooten et al., 1988 ), a comprehensive discharge planning protocol ( Kennedy et al., 1987 ; Naylor, 1990; Naylor et al., 1994 ), a (patient- or nurse-initiated) telephone call-back system ( Young, 1990; Siegel, 1992; Wachter, 1995; Shu et al., 1996 ), hospital discharge planning staff ( Mamon et al., 1992 ; Peters et al., 1997 ), and home visits by community nurses ( Van Harteveld et al., 1997 ).

In order to determine what kind of intervention could prevent postdischarge problems on a participating ophthalmic unit, studies comparing the effectiveness of interventions are needed. Unfortunately, these studies are scarce, and those that are available do not recommend any specific intervention. For example, Barnason \& Zimmerman (1995) compared three teaching programmes (an inpatient teaching programme, a postdischarge telephone follow-up programme and a postdischarge group teaching programme) and found similar patient teaching outcomes regardless of the type of teaching programme the patients were involved in. In another study, two systems of telephone follow-up (a nurse-initiated and a patient-initiated telephone programme) were compared with a third group of patients receiving no intervention. Although no differences were found in patient satisfaction with the health education, the results suggested that patients are unlikely actively to seek the information they need ( Bostrom et al., 1996 ). In conclusion, not for scientific but for pragmatic reasons, we initiated a community-orientated, nurse-initiated Telephone Reassurance Programme (TRP) during which patients who had recently been discharged were phoned at home. The most important pragmatic reasons were phoned this method is relatively cheap, patients do not have to come to the hospital (e.g. for teaching) and patients can be telephoned from the ward during quiet periods.

Studies concerning nurse-initiated TRPs often only describe the problems mentioned by patients or the interventions subsequently applied by the nurse who made the telephone call. The problems patients mention during a call are, for instance, health problems and social problems ( Bowman et al., 1994 ), problems concerning safety and medication ( Closson et al., 1994 ), and difficulty in accepting their changed health status, concern about financial difficulties and uncertainty ( Keeling \& Dennison, 1995). Nursing interventions subsequently applied by nurses are, for example, guidance and support ( Cave, 1989; Orticio \& Swan, 1992; Closson et al., 1994 ; Keeling \& Dennison, 1995; Turner, 1996), instruction ( Shesser et al., 1986 ; Orticio \& Swan, 1992; Keeling \& Dennison, 1995), referral to a physician or other caregivers ( Shesser et al., 1986 ; Orticio \& Swan, 1992; Keeling \& Dennison, 1995) and teaching ( Cave, 1989; Phillips, 1993).

In a few studies the effectiveness of a nurse-initiated TRP has been tested. Positive effects were found on patients' knowledge of the disease, recommended exercises and all teaching areas but no effects were found on knowledge of diet, medication, restrictions in physical activity or rest ( Garding et al., 1988 ). In another study, male patients who received a follow-up telephone call had a more positive perception of their visit to the Emergency Department than male patients who did not participate in the TRP ( Shesser et al., 1986 ). Finally, Phillips (1993) found no difference on the variable 'quality of life' between patients who were called and those who were not.

In conclusion, little is known about the effect of a nurse-initiated TRP on postdischarge problems, so we decided to study this effect. We also wanted to study both the short-term and the long-term effects of the TRP.

The objective of this study was to answer the following research question: what is the effect of a nurseinitiated TRP on postdischarge problems reported by recently discharged ophthalmic patients?

\section{METHOD}

\section{INTERVENTION}

Patients in the intervention group were phoned 3-6 days after discharge by an experienced nurse. Before calling, the nurse went through a structured form containing relevant information about the patient's admission and discharge conditions (e.g. living situation, ophthalmic diagnoses and treatment, and description of the treated eye). These forms were prepared during hospitalization for all included patients. 
During the call, the nurse used a structured interview schedule which was attached to the form. The interview schedule covered 10 aspects (see Appendix). Examples of these aspects are: 'How is your treated eye?' and 'Is there anything else you would like to know about your treatment?'. All aspects included in the interview schedule were discussed with the patient, if relevant. If a patient mentioned a problem or asked a question, the nurse could compare this with the information on the form and intervene. In decreasing order, giving reassurance, referring to a medical doctor, and giving information, advice or instructions were the most frequently applied nursing interventions ( Boter et al., 1998a ).

To make effects of the intervention more likely to be the results of the intervention itself than of the personal contribution of a single nurse, six nurses participated in the project.

\section{DESIGN}

A randomized clinical trial was carried out with a post-test only design ( Fig. 1). The two times of measurement were 7 days $\left(T_{1}\right)$ and 30 days $\left(T_{2}\right)$ after discharge. The second time of measurement, 30 days after discharge, was chosen because we still expected to find a smaller but significant effect at that time. Patients in the intervention group participated in the TRP, in addition to receiving conventional discharge treatment. Patients in the control group received only the conventional discharge treatment.

\section{[FIGURE 1]}

\section{SUBJECTS}

The study included Dutch-speaking, adult ophthalmic patients who had been treated and hospitalized for at least two days in the period from March to November 1997 on the participating ophthalmic ward of the University Hospital Vrije Universiteit in Amsterdam, the Netherlands. Patients were excluded if they were admitted from another ward or care institute before they were hospitalized, or were discharged to institutional care. Patients with no telephone, or those who were not able to answer the telephone (for example because of hearing problems) and had no one else to answer it, were also excluded.

\section{PROCEDURES}

Approval was obtained from the ethics review board and two scientific committees of the study hospital before starting recruitment. Patients who met the inclusion criteria were informed about the project and invited to participate. All patients were provided with the routine information and conventional care before and on discharge. Immediately after discharge, patients from whom consent was obtained were randomized to the intervention or control group by an independent researcher. Patients in the intervention group received a letter signed by the head nurse, informing them about the telephone call. Patients were sent a questionnaire 5 or 6 days after discharge and an identical questionnaire was sent on the 28th day. If the successive questionnaires were not returned before the 11th day or the 35th day after discharge, respectively, the researcher reminded patients by telephone. It should be noted that patients in the intervention group only received the 7th-day questionnaire if they were phoned in time, and patients in both groups only received the 30th-day questionnaire if the 7th-day questionnaire was returned within 14 days after discharge.

\section{OUTCOMES}

To cover a broad range of (potential) postdischarge problems, the following patient outcomes were measured: 'Informational needs', 'Uncertainty', 'Emotional complaints' and 'Functional limitations'.

'Informational needs', i.e. the patient's perception of not being sufficiently informed, was measured by means of a newly developed instrument. The instrument included 39 items and the content was based on the Patient Learning Need Scale (PLNS: Bubela et al., 1990 ). It is a general instrument that can be used with divergent patient populations. Factor analysis indicated a strong central factor suggesting a unidimensional scale. Examples of the items included are: 'Last week, did you need more information about: when you can take a bath or shower?' or 'How you can prevent a complication from occurring?'. Respondents could indicate by answering ' $0=$ no' or ' $1=$ yes' according to whether or not they felt adequately informed. The scale yields a total score with a minimum score of zero (no informational needs) and a maximum score of 39 (maximum amount of informational needs). Cronbach's alpha in the present study was 0.95 .

'Uncertainty', i.e. the patient's inability to determine the meaning of illness-related events, was measured by means of a translated version of the Mishel Uncertainty in Illness Scale - Community form (MUIS-C: Mishel, 1981). This instrument is used for patients who are not hospitalized and are not likely to be 
receiving medical intervention (Mishel, 1990). This one-factor scale includes 23 items and is measured on a five-point scale ranging from 'strongly disagree' to 'strongly agree', with items worded in both directions. The total scores for 'Uncertainty' can range from 23 (low uncertainty) to 115 (high uncertainty). The MUIS-C is based on the MUIS, which has been validated for both divergent and convergent validity and has proved to be very sensitive to differences in clinical population samples ( Mishel, 1990). Cronbach's alpha in the present study was 0.91 .

'Emotional complaints' were measured by means of a dimension of the Problems After Discharge Questionnaire (PADQ: Mistiaen et al., 1997 ). This dimension is based on a five-point scale, varying from ' $1=$ no trouble at all' to '5 = very much trouble'. It includes six items related to a patient's feelings: lonely, restless, sad, anxious, uncertain and worried. The mean scores can range from 1 to 5. Cronbach's alpha in the present study was 0.91 .

'Functional limitations' were measured by means of another dimension of the PADQ. It was used to assess the problems respondents perceived in performing daily activities independently. The dimension was scored on a five-point scale, ranging from ' $1=$ no difficulty at all' to ' $5=$ not able to'. It covers three areas: personal care (five items - washing hands, taking a bath or shower, dressing, combing hair, and eating and drinking), with a Cronbach's alpha of 0.80 in the present study; housekeeping (seven items - preparing meals, shopping, heavy housework, laundry, making beds, washing dishes, light housework), with a Cronbach's alpha of 0.94 ; and mobility (five items - getting out of bed, going to the toilet, climbing stairs, walking outdoors, travelling), with a Cronbach's $\alpha$ of 0.81 . All mean scores can range from 1 to 5 . The dimensions 'Emotional complaints' and 'Functional limitations' were developed by Mistiaen et al. (1997 ), who used the dimensions in an earlier study on postdischarge problems. Both dimensions were assessed for content validity ( Mistiaen et al., 1997 ). To find evidence of criterion-related validity, the dimensions were assessed against the BRASS-index. The aim of the BRASS-index is to identify, shortly after admission, those patients who are at risk for prolonged hospitalization and are in need of discharge planning resources in order to reduce or avoid post-discharge problems ( Blaylock \& Cason, 1992). Tests revealed that both dimensions correlated, as was expected ( Mistiaenet al. submitted).

The four outcomes were combined in a postal questionnaire. It was pilot-tested on five hospitalized ophthalmic patients, after which amendments (e.g. on readability) were made. Subsequently, for a period of one month, the whole procedure (including the intervention and the postal questionnaires) was pilot-tested on patients who met the inclusion criteria $(\mathrm{n}=79)$, after which the form, interview schedule and questionnaire were again improved.

\section{DATA ANALYSIS}

Independent-Samples t-tests (on 'Uncertainty') and Mann-Whitney U-tests (on the other, not normally distributed, outcomes) were used to determine the differences between the intervention and the control group. The Statistical Package for the Social Sciences (SPSS) was applied for all tests, using a significance level of 0.05 (two-tailed).

\section{RESULTS}

\section{PARTICIPANTS}

During the research period, 559 patients were admitted. In all, 134 patients were excluded because, for example, they had been discharged to institutional care $(n=46)$, admitted from or transferred to another unit (e.g. Emergency Department; $n=41)$, or they had received no medical treatment $(n=15)$.

Among the 425 patients who met the inclusion criteria, 31 patients were unwilling to participate. Those who were unwilling to participate and the remaining 394 patients did not differ with respect to age, gender or length of hospital stay. A total of 196 patients was randomly assigned to the intervention group and 198 to the control group. In the intervention group, 183 patients were phoned in time (mean score 3.9 days after discharge; mode three days). Patients who were phoned too late, or were not contacted at all, were younger than those who were phoned in time: 55.1 (SD 16.1) years vs 65.7 (SD 16.6), $\mathrm{P}<0.05$. No differences were found on the variables gender or length of hospital stay.

Subsequently, the 7th-day questionnaire was completed by 143 patients in the intervention group and 154 patients in the control group, within 14 days after discharge (response $78 \%$ in both groups). Reasons for non-response to the first questionnaire were: 'not feeling like completing a questionnaire' (16\%), 'feeling too ill' (9\%), 'having no problems' (7\%), 'being readmitted' (5\%), 'other' (11\%) and 'unknown' (52\%). 
Within 42 days after discharge, 113 patients in the intervention group and 127 patients in the control group completed the 30th-day questionnaire (response $62 \%$ and $64 \%$, respectively). Reasons for non-response to the second questionnaire were: 'feeling too ill' (10\%), 'being readmitted' (10\%), 'having no problems' (6\%), 'not feeling like completing a questionnaire' (4\%), 'other' (23\%) and 'unknown' (46\%). Patients who responded to both questionnaires differed on the outcomes from those who returned only the 7 th-day questionnaire. It was found that respondents in the intervention group who completed the 7 th-day but not the 30th-day questionnaire scored significantly higher on all 7th-day outcome measures than patients in the same group who returned both questionnaires. This indicates that patients in the intervention group who returned only the 7th-day questionnaire had more problems 1 week after discharge than those who returned both questionnaires. Among patients in the control group the same difference was found on 'Uncertainty'.

The mean time-lapse between discharge and completion of the 7th- and 30th-day questionnaires was 8.1 days $(\mathrm{SD}=2.4)$ and 31.8 days $(\mathrm{SD}=3.2)$, respectively.

The 297 respondents to the 7th-day questionnaire and 84 non-respondents did not differ on the variables age, gender or length of hospital stay. Furthermore, the 240 respondents and 154 non-respondents to the 30th-day questionnaire did not differ on the variables age or length of hospital stay. However, men more often completed the questionnaire than women $(\mathrm{P}<0.01)$.

General baseline characteristics of the respondents are shown in Table 1. No differences were found between the respondents of the intervention and control groups in relation to these characteristics. However, patients in the intervention group who responded to the 30th-day questionnaire had a longer hospital stay than those in the control group: 4.1 (SD 2.5) days vs 3.5 (SD 2.2), $\mathrm{P}<0.05$. When analyzing the results, 'length of hospital stay' was not used as a covariate because it had low correlations with the outcomes (range 0.06-0.17).

Most patients were treated for cataract (43\%), glaucoma (15\%), retina disorders (14\%), or cornea disorders $(13 \%)$.

\section{IMPACT OF THE INTERVENTION}

No differences were found on the outcomes between patients in the intervention group and those in the control group 1 week after discharge (Table 2).

Again 30 days after discharge no differences were found, except for housekeeping. Patients in the intervention group had fewer problems with housekeeping than patients in the control group: 1.40 (SD 0.78 ) vs 1.69 (SD 1.09), $\mathrm{P}<0.05$.

Though practically no differences were found between the two groups, the TRP might have had an influence on the degree to which the outcome scores declined during the first month after discharge (Table 3). However, here again no differences were found between the results of the patients who participated in the TRP and those who did not.

\section{SUBGROUP ANALYSES}

Subgroup analyses were carried out in order to determine whether the TRP helped to reduce the postdischarge problems of specific patient groups. For example, the outcomes of male patients in the intervention group were compared with those of male patients in the control group. However, no effects of the intervention were found when comparisons were made with regard to gender, age, length of hospital stay, ophthalmic diagnoses, living situation and history of admission.

\section{[TABLE 1][TABLE 2][TABLE 3]}

\section{DISCUSSION}

A randomized clinical trial was carried out to evaluate a nurse-initiated Telephone Reassurance Programme for ophthalmic patients who had recently been discharged. Seven and 30 days after discharge, outcomes (Informational needs, Uncertainty, Emotional complaints and Functional limitations) revealed no differences between patients who participated in the programme and patients who did not, except for housekeeping. Patients in the intervention group had fewer problems with housekeeping 30 days after discharge. Nevertheless, the clinical relevance of this significant difference is minimal, and it might also be the result of a Type I error. The results of the present study are partially supported by the findings of other studies on the effect of this type of programme. Garding et al. (1988) found a few positive effects, namely on the patient's knowledge of the disease, recommended exercises, and all teaching areas together. Shesser 
Boter, H., Mistiaen, P., Groenewegen, I. A randomized trial of a telephone reassurance programme for patients recently discharged from an ophthalmic unit. Journal of Clinical Nursing: 2000, 9(2), 199-206

et al. (1986 ) reported that only male patients evaluated their visit to the Emergency Department in combination with the TRP more positively, compared to men who did not participate in the TRP. No effects were found by Phillips (1993) on 'quality of life'.

Since no effects were found in the present study, it is important to discuss the limitations of the study before it is concluded that, in general, a TRP does not reduce post-discharge problems.

Firstly, some points are made about the participants. To be included in the study, patients had to be alert and admitted from and discharged to their home. On these grounds, some of the patients who might have benefited most from the TRP were excluded, for instance those who came from an Emergency Department. In addition, other categories of patients who have had more serious treatment (e.g. heart surgery or chemotherapy) might have benefited more from the TRP. Finally, bias is introduced by the fact that the patients who responded to both questionnaires differed on the outcomes from those who returned only the 7th-day questionnaire.

The outcomes measured may also be a limitation of the study. Due to a general lack of theoretical knowledge about the prevention of postdischarge problems by introducing a TRP, it is possible that the TRP affects outcomes other than those measured. Additionally, it is possible that the instruments used were not sensitive enough to detect small differences between the two groups. For example, 'Informational needs' was not measured with an instrument that was specifically developed for ophthalmic patients and we found some floor-effects on 'Emotional complaints', 'Personal care', and 'Mobility'. Furthermore, we did not compare the groups on a variable such as 'stressful life events' or 'coping'. Other than hospitalization, major changes in a patient's life might influence the possible effects of the TRP. Furthermore, the intervention might have had an adverse effect on 'Uncertainty' and 'Emotional complaints' for some patients. For instance, the coping strategy 'denial' is used by some patients to deny the situation they are in, and the TRP might have reminded them of their problems, thus increasing their worries and distress.

The intervention itself might also be a reason why no effects were found in favour of the TRP. The 'intervention dose' might have been too low to achieve the intended outcomes. The nurses were not specifically trained in telephone assistance. The main reason for making this decision was that we wanted to make the programme easily applicable for other units, and only experienced nurses phoned the patients at home.

In addition, a few more comments should be made about the intervention. The lack of training might have resulted in nurse-related differences in the content and quality of the telephone calls. However, the nurses discussed their telephone calls and informed each other about the postdischarge problems mentioned by patients and the interventions they subsequently applied. Secondly, the analysis revealed no differences between the outcomes of patient groups who were phoned by different nurses. Another potential problem was that the intervention was not stable: the knowledge nurses had about post-discharge problems increased during the period in which they phoned the patients. Patients in the intervention group who were included in the study in October or November 1997 were phoned by nurses who had more experience with the TRP than patients in the same group who were discharged in March or April 1997. Here again, however, the analysis revealed no differences in the outcomes between patients who were called early in the study and those who were called later. Finally, nurses who called the patients after discharge discussed their TRPrelated experiences with other nurses from the unit. It is possible that during the study all nurses from the unit paid increased attention to the prevention of post-discharge problems by preparing all admitted patients more adequately before discharge. However, this also showed no trend.

Despite the lack of significant results, a positive side-effect of the TRP was that, as nurses reported, future patients might benefit from the increasing knowledge nurses have about postdischarge problems and the improvements they made, for example, in their teaching strategies. In addition, patients in the intervention group appreciated the TRP. For instance, $89 \%$ of the patients wanted to be called again after hospitalization and only $6 \%$ reported that they considered the call to be unnecessary ( Boter et al., 1998a ).

We recommend that in future research the variable 'stressful life events' should be included as a possible modifier of the TRP's impact. Additional research is also needed to assess the contribution of the TRP in decreasing the use of healthcare services or improving satisfaction with the care provided. Furthermore, we suggest that the effect of the TRP on patient outcomes should be studied among other more critically ill, patient groups. 
Boter, H., Mistiaen, P., Groenewegen, I. A randomized trial of a telephone reassurance programme for patients recently discharged from an ophthalmic unit. Journal of Clinical Nursing: 2000, 9(2), 199-206

\section{ACKNOWLEDGEMENTS}

The authors wish to acknowledge the contribution to the research made by the nursing staff: L. Meijers, L. van Voorthuizen, L. van Thol, J. Jansen and J. Schutte. We also thank A. Veeger (staff member, Nursing Development Office) and A. Zeegers (researcher) for their help.

\section{FOOTNOTES}

\section{INFORMATION POINT:}

Type I and Type II errors

\section{REFERENCES}

Allen M., Knight C., Falk C. \& Strang V. (1992) Effectiveness of a preoperative teaching programme for cataract patients. Journal of Advanced Nursing 17, 303-309.

Allen M. \& Oberle K. (1993) Follow-up of day-surgery cataract patients. Journal of Ophthalmic Nursing and Technology 12, 211- 216.

Arenth L.M. \& Mamon J.A. (1985) Determining patient needs after discharge. Nursing Management 16, 2024.

Barnason S. \& Zimmerman L. (1995) A comparison of patient teaching outcomes among postoperative coronary artery bypass graft (CABG) patients. Progress in Cardiovascular Nursing 10(4), 11-20.

Blaylock A. \& Cason C.L. (1992) Discharge planning predicting patients' needs. Journal of Gerontological Nursing 18(7), 5-10.

Bostrom J., Caldwell J., McGuire K. \& Everson D. (1996) Telephone follow-up after discharge from the hospital: does it make a difference? Applied Nursing Research 9(2), 47-52.

Boter H., Groenewegen I., Van Dijk L. \& Mistiaen P. (1998a) Telefonische nazorg voor oogheelkunde patieĖnten (Aftercare by telephone for ophthalmic patients). TVz 108, 288-291.

Boter H., Mistiaen P., Duijnhouwer E. \& Groenewegen I. (1998b) The problems of elderly patients at home after ophthalmic treatment. Journal of Ophthalmic Nursing and Technology 17, 59-65.

Bowman G.S., Howden J., Allen S., Webster R.A. \& Thompson D.R. (1994) A telephone survey of medical patients 1 week after discharge from hospital. Journal of Clinical Nursing 3, 369-373.

Boyle K., Nance J. \& Passau-Buck S. (1992) Post-hospitalization concerns of medical-surgical patients. Applied Nursing Research 5, 122-126.

Brooten D., Brown L.P., Munro B.H. et al. (1988) Early discharge and specialist transitional care. Image the Journal of Nursing Scholarship 20, 64-68.

Bubela N., Galloway S., McCay E. et al. (1990) The Patient Learning Needs Scale: reliability and validity. Journal of Advanced Nursing 15, 1181-1187.

Cave L.A. (1989) Follow-up phone calls after discharge. American Journal of Nursing 89, 942-943.

Closson B.L., Mattingly L.J., Finne K.M. \& Larson J.A. (1994) Telephone follow-up program evaluation: application of Orem's self-care model. Rehabilitation Nursing 19, 287-292.

Garding B.S., Kerr J.C. \& Bay K. (1988) Effectiveness of a program of information and support for myocardial infarction patients recovering at home. Heart and Lung 17, 355-362.

Keeling A.W. \& Dennison P.D. (1995) Nurse-initiated telephone follow-up after acute myocardial infarction: a pilot study. Heart and Lung 24, 45-49.

Kennedy L., Neidlinger S. \& Scroggins K. (1987) Effective comprehensive discharge planning for hospitalized elderly. Gerontologist 27, 577-580.

Law M.L. (1997) A telephone survey of day-surgery eye patients. Journal of Advanced Nursing 25, 355363.

McWilliam C.L. \& Sangster J.F. (1994) Managing patient discharge to home: the challenges of achieving quality of care. International Journal for Quality in Health Care 6, 147-161.

Mamon J., Steinwachs D.M., Fahey M., Bone L.R., Oktay J. \& Klein L. (1992) Impact of hospital discharge planning on meeting patient needs after returning home. Health Services Research 27, 155-175.

Mishel M.H. (1981) The measurement of uncertainty in illness. Nursing Research 30, 258-263.

Mishel M.H. (1990) Uncertainty in Illness Scales. (Manual) 3 University of North Carolina, Chapel Hill, USA.

Mistiaen P., Duijnhouwer E., Prins-Hoekstra A., Ros W. \& Blaylock A. (Submitted) Predictive validity of the BRASS-index in screening patients with post-discharge problems. Journal of Advanced Nursing 30(5).

Mistiaen P., Duijnhouwer E., Wijkel D., De Bont M. \& Veeger A. (1997) The problems of elderly people at home one week after discharge from an acute care setting. Journal of Advanced Nursing 25, 1233-1240. 
Boter, H., Mistiaen, P., Groenewegen, I. A randomized trial of a telephone reassurance programme for patients recently discharged from an ophthalmic unit. Journal of Clinical Nursing: 2000, 9(2), 199-206

Naylor M.D. (1990) Comprehensive discharge planning for hospitalized elderly: a pilot study. Nursing Research 39, 156-161.

Naylor M., Brooten D., Jones R., Lavizzo-Mourey R., Mezey M. \& Pauly M. (1994) Comprehensive discharge planning for the hospitalized elderly. A randomized clinical trial. Annals of Internal Medicine 120, 999-1006.

North M., Meeusen M. \& Hollinsworth P. (1991) Discharge planning: increasing client and nurse satisfaction. Rehabilitation Nursing 16, 327-329.

Orticio L.P. \& Swan J. (1992) Implementation of the postdischarge follow-up call in the patient care units. Insight 17(2), 15-19.

Peters P., Fleuren M. \& Wijkel D. (1997) The quality of the discharge planning process: the effect of a liaison nurse. International Journal for Quality in Health Care 9, 283-287.

Phillips C.Y. (1993) Postdischarge follow-up care: effect on patient outcomes. Journal of Nursing Care Quality 7(4), 64-72.

Shesser R., Smith M., Adams S., Walls R. \& Paxton M. (1986) The effectiveness of an organized emergency department follow-up system. Annals of Emergency Medicine 15, 911-915.

Shu E., Mirmina Z. \& Nystrom K. (1996) A telephone reassurance program for elderly home care clients after discharge. Home Healthcare Nurse 14, 154-161.

Siegel S.B. (1992) Telephone follow-up programs as creative nursing interventions. Pediatric Nursing 18(1), 86-89.

Smith S.J. \& Drance S.M. (1984) Dif $($ culties patients have at home after cataract surgery. Canadian Journal of Ophthalmology 19(1), 6-9.

Tierney A., Worth A., Closs S.J., King C. \& Macmillan M. (1994) Older patients' experiences of discharge from hospital. Nursing Times 90(21), 36-39.

Turner D. (1996) Can telephone follow-up improve post-discharge outcomes? British Journal of Nursing 5, 1361-1365.

Van Beelen A. (1996) Nog even een telefoontje (Just a minute, I have to make a phone call). Verpleegkunde Nieuws 10(2), 11-12.

Van Harteveld J.T., Mistiaen P.J. \& Dukkers van Emden D.M. (1997) Home visits by community nurses for cancer patients after discharge from hospital: an evaluation study of the continuity visit. Cancer Nursing 20(2), 105-114.

Wachter C.E. (1995) Triage of medical or social issues through preoperative and postoperative telephone calls by primary nurses. Insight 20(2), 16-20.

Young C.M. (1990) The postoperative follow-up phone call: an essential part of the ambulatory surgery nurse's job. Journal of Post Anesthesia Nursing 5, 273-275.

\section{FIGURES}

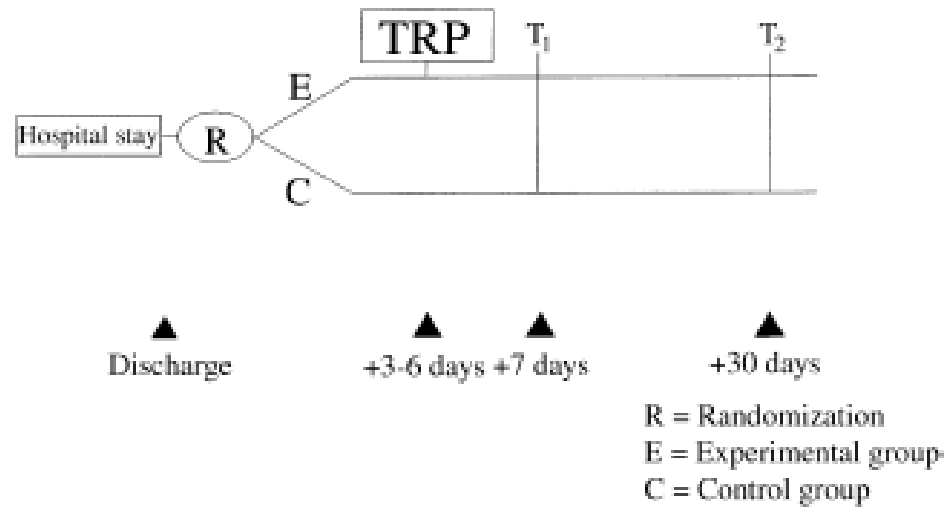

Figure 1 Trial design. 
Boter, H., Mistiaen, P., Groenewegen, I. A randomized trial of a telephone reassurance programme for patients recently discharged from an ophthalmic unit. Journal of Clinical Nursing: 2000, 9(2), 199-206

\section{TABLES}

Table 1 Baseline characteristics of respondents to the 7 th-day questionnaire

\begin{tabular}{ll}
\hline & $\begin{array}{l}\text { All patients } \\
(n=297)\end{array}$ \\
\hline Sociodemographic characteristics & \\
Mean age (SD) & $66.6(16.1)$ \\
Female (\%) & 56.9 \\
Living alone (\%) & 38.2 \\
Mean length of hospital stay (SD) & $3.9(2.9)$ \\
Admission(s) in previous two years for & 35.2 \\
$\quad$ ophthalmic treatment (\%) & 92.9 \\
Self-supporting in ADL and/or IADL (\%) & 14.1 \\
Having home help and/or home nursing (\%) & \\
\hline
\end{tabular}

Table 2 Comparison of mean scores between the intervention group and the control group on the 7 th-day questionnaire

\begin{tabular}{|c|c|c|c|c|c|}
\hline & \multirow{2}{*}{$\begin{array}{l}\text { Theoretical } \\
\text { range }\end{array}$} & \multicolumn{2}{|c|}{$\begin{array}{l}\text { Intervention } \\
(n=143)\end{array}$} & \multicolumn{2}{|c|}{$\begin{array}{l}\text { Control } \\
(n=154)\end{array}$} \\
\hline & & Mean & SD & Mean & SD \\
\hline In formational needs & $0-39$ & 4.59 & 6.69 & 5.35 & 7.34 \\
\hline Uncertainty & $23-115$ & 49.42 & 14.42 & 49.93 & 15.81 \\
\hline Emotional complaints & $1-5$ & 1.70 & 0.81 & 1.64 & 0.81 \\
\hline \multicolumn{6}{|l|}{ Functional limitations } \\
\hline Personal care & $1-5$ & 1.24 & 0.54 & 1.32 & 0.65 \\
\hline Housekeeping & $1-5$ & 2.31 & 1.22 & 2.42 & 1.31 \\
\hline Mobility & $1-5$ & 1.65 & 0.82 & 1.80 & 0.96 \\
\hline
\end{tabular}

Note: the higher the score, the more problems.

Table 3 Decline of mean scores between 1 week and 1 month after discharge

\begin{tabular}{|c|c|c|c|c|}
\hline & \multicolumn{4}{|c|}{$\begin{array}{l}\text { 7th-day questionnaire minus } \\
\text { 30th-day questionnaire }\end{array}$} \\
\hline & \multicolumn{2}{|c|}{$\begin{array}{l}\text { Intervention } \\
(n=113)\end{array}$} & \multicolumn{2}{|c|}{$\begin{array}{l}\text { Control } \\
(n=127)\end{array}$} \\
\hline & Mean & SD & Mean & SD \\
\hline Informational needs & 0.41 & 5.61 & 2.17 & 5.12 \\
\hline Uncertainty & 1.91 & 11.16 & 1.15 & 9.38 \\
\hline Emotional complaints & 0.14 & 0.53 & 0.18 & 0.60 \\
\hline \multicolumn{5}{|l|}{ Functional limitations } \\
\hline Personal care & 0.11 & 0.42 & 0.12 & 0.32 \\
\hline Housekeeping & 0.66 & 0.77 & 0.63 & 0.84 \\
\hline Mobility & 0.21 & 0.49 & 0.18 & 0.46 \\
\hline
\end{tabular}


Boter, H., Mistiaen, P., Groenewegen, I. A randomized trial of a telephone reassurance programme for patients recently discharged from an ophthalmic unit. Journal of Clinical Nursing: 2000, 9(2), 199-206

\section{APPENDIX}

\section{Appendix}

Interview schedule used during the intervention.

1. How are you?

2. How is your treated eye?

3. How are you coping with cleaning your eye, putting in eyedrops/using ointment?

4. How are you coping with the following instructions? - not to rub the treated eye, bend down or lift;

- to wear an eye shield at bedtime.

5. How are you coping with performing self-care tasks and running your household?

6. How is the home nursing or home help you receive? (If relevant)

7. Is there anything else you would like to know about your treatment?

8. Would you like to know more about this? (If the patient thinks the disease or treatment will affect his/her life.)

9. Do you know what you can do if you need more in formation?

10. Is there anything else you would like to ask or discuss? 